The extended lattice symmetry of the rich and varied structure of solid $\mathrm{C}_{60}$ matches the aesthetic appeal of molecular $\mathrm{C}_{60}$, making $\mathrm{C}_{60}$ arguably Nature's most elegant example of crystalline symmetry.

\section{Squaring the Circle}

\section{W.I.F. David}

\author{
ISIS Science Division \\ Rutherford Appleton Laboratory, Chilton, UK.
}

Despite its relatively recent discovery, carbon-60 is, along with benzene and methane, probably the best known carbonbased molecule. $\mathrm{C}_{60}$ is the most easily synthesized and abundant example of the carbon-cage molecular compounds collectively known as the fullerenes. Excellent work has been performed on the higher fullerenes, particularly $\mathrm{C}_{70}$. We shall, however, focus on $\mathrm{C}_{60}$. Although the most stable carboncage structure, it is also, perhaps, the most unusual. The majority of fullerenes adopt a relatively low point-group symmetry. In contrast to this, $\mathrm{C}_{60}$ with perfect icosahedral symmetry, adopts the very highest. It forms a truncated icsoahedron - a molecular soccer ball - formed from twelve pentagonal and twenty hexagonal faces. This was first confirmed by solution NMR measurements $[1,2]$ which showed a sharp single resonance in the ${ }^{13} \mathrm{C}$ NMR spectrum indicating that all carbon atoms in $\mathrm{C}_{60}$ are equivalent.

While all 60 carbon atoms are identical, there are two distinct types of bonds. Each carbon atom is bonded to three nearest neighbours by two longer $(\approx 1.45 \AA)$ and one shorter $(\approx 1.40 \AA)$ bond. These two bonds have a differing bond order and although not of pure single $(\approx 1.54 \AA)$ and double $(\approx 1.32$ A) character are extensively discussed as such in the fullerene literature. The "double" bonds are inter-pentagonal and lead to a sequential "single"-"double" bond character around the hexagonal face. This is again consistent with icosahedral symmetry which demands that the hexagons are not of sixfold but only of three-fold symmetry. There is thus bond localization around the hexagons in $\mathrm{C}_{60}$ and, as a consequence, the hexagons are chemically different from a benzene ring and, more importantly, distinct from the carbon hexagons that constitute the layers of the graphite structure. This indicates that important and subtle differences exist between the inter-layer bonding in graphite, which is of a van der Waals character, and the inter-molecular interaction in $\mathrm{C}_{60}$. Indeed the bonding anisotropy in $\mathrm{C}_{60}$ plays a crucial rôle in the reorientational molecular motion, and consequently, in the rich structural variation of crystalline $\mathrm{C}_{60}$ as a function of temperature that currently challenges first-principles calculations of the crystal structure.

\section{The Ideal Molecular Bubble Raft}

One of the problems facing the crystallo-

W.I.F. David is a senior scientist with the ISIS Science Division and a professor at University College, London. admitted within the context of quasicrystals. $\mathrm{C}_{60}$ is, however, not quasicrystalline. At room temperature, it circumvents the problem posed by five-fold symmetry by rapid reorientation in an orientationally ordered phase. A number of techniques have shown that $\mathrm{C}_{60}$ is hopping from one orientation to another on the time-scale of around 9 ps. This is extremely rapid for a molecule with a considerable moment of inertia. Indeed, a simple calculation shows that unhindered rotation at $300 \mathrm{~K}$ leads to a characteristic time of 3 ps. Moreover, dissolving $\mathrm{C}_{60}$ in solution results in a reorientation time that is $50 \%$ slower than in the solid!

The molecular environment in disordered solid $\mathrm{C}_{60}$ at room temperature is extremely conducive to reorientation. NMR measurements [2] indicate the activation energy for reorientation is some $690 \mathrm{~K}$. At room temperature, therefore, orientations at the top of the activation energy barrier are significantly populated at some $10 \%$ of the value of the minimum energy configurations. The scattering density is thus highly isotropic and can be accurately modelled by a simple isotopic shell of scattering. The existence of reorientational hopping and an activation energy, however, necessitates deviations from isotropic behaviour which have indeed

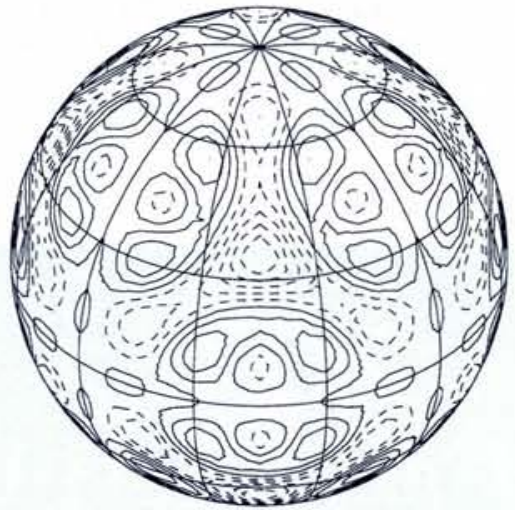

been observed in a careful single-crysta $\mathrm{X}$-ray synchrotron radiation experiment [3] It may be shown that the structure factor associated with a $\mathrm{C}_{60}$ "bucky-ball" modelled as a spherical shell of scattering of radius $R$ is given by

$$
F(\boldsymbol{h})=\sqrt{4 \pi} f_{\mathrm{c}}(\boldsymbol{h}) j_{0}(Q R) C_{0,1}
$$

where $f_{c}(\boldsymbol{h})$ is the $\mathrm{X}$-ray/neutron scattering factor for carbon that includes thermal motion effects, $Q=|Q(h)|=(4 \pi \sin \theta) / \lambda$ is the wave-vector magnitude, $j_{0}(Q R)=\sin (Q R) /$ $(Q R)$ is the zeroth-order spherical Bessel function, and $C_{0,1}$ is the zeroth-order coefficient to be determined by refinement. Variations from isotropy may be accommodated by introducing higher order surface harmonics that are consistent with both point group and molecular symmetry. The resulting structure factor equation is

$$
F(\boldsymbol{h})=\sqrt{4 \pi} f_{\mathrm{c}}(\boldsymbol{h}) \sum_{l \mathrm{v}} \sum^{-1} j_{j}\{Q(\boldsymbol{h}) R\} C_{l, v} S_{l}^{v}(\boldsymbol{h})
$$

where $/$ is the order of the surface harmonic and $v$ discriminates between different surface harmonics $S_{j}^{v}(h)$ of the same order The refined coefficients from the single crystal experiment along with a more recent powder diffraction analysis [4] determine time derivations from isotropy. Perhaps surprisingly, maximum scattering density occurs close to the [110] intermolecular bonding directions. Minimum scattering density is along [111] directions (Fig. 1). The maximum and minimum deviations from isotropy are nevertheless small, of order $+10 \%$ and $-16 \%$, respectively. This agrees, heuristically with the relatively low activation energy of $690 \mathrm{~K}$ which implies that there is significant population at both the bottom and the top of the activation energy barrier.

\section{Low-Temperature Structure}

Differential scanning calorimetry [5] first revealed that solid $\mathrm{C}_{60}$ undergoes a structural phase transition at around $260 \mathrm{~K}$. The resulting diffraction pattern no longer conforms to a face-centred cubic lattice with the octahedral space group $\mathrm{Fm} \overline{3} \mathrm{~m}$ associated with ideal close-packed spheres representing the orientationally disordered $C_{60}$ mole-

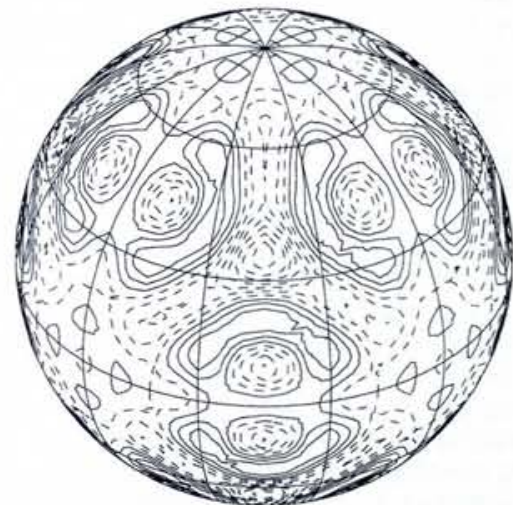

Fig. 1 - There is good agreement between the orientation distribution functions, viewed down one of the $<111>$ directions, for the disordered $C_{60}$ phase at $270 \mathrm{~K}$ obtained from single crystal $X$-ray synchrotron data (a, left) [3] and by high-resolution neutron powder diffractometry (b, right) [4]. Contours in a) show a density deficit of $-16 \%$ at $<111>$ and a density excess of $9 \%$ close to $\langle 110\rangle$, where the scattering density, $\rho(\theta, \phi)=4 \pi \Sigma \Sigma_{v} C_{l,} S_{\rangle}^{v}(\theta, \phi)$ is calculated from the refined coefficients $C_{l, v}$ up to $I=18$ and omitting $I=0$ to emphasize the deviations from spherical symmetry; full and dotted lines correspond to positive and negative contours, respectively. For (b), coefficients up to $I=18$ were measured and the $I=0$ coefficient was suppressed to emphasize the deviations from spherical symmetry. 

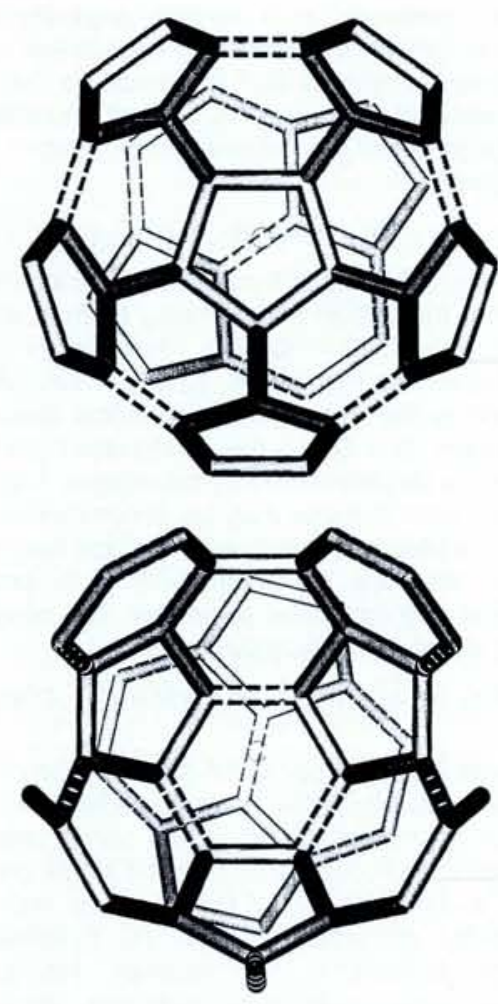

cules The regular spacing of Bragg peaks, however, remains and indicates that the crystal structure is now primitive cubic. The molecule's centres are, nevertheless, still located at the face-centred cubic positions. The primitive cubic symmetry arises because the $C_{60}$ molecules at $(000),(1 / 21 / 20)$, $(1 / 201 / 2)$ and $(01 / 21 / 2)$ are in four distinct but symmetry related orientations governed by the space group $\mathrm{Pa} \overline{3}$. This apparently small reduction in symmetry does in fact have a profound effect on the $\mathrm{C}_{60}$ molecular site symmetry which reduces from cubic,

Fig. 3-A representation of the reorientation mechanism in the ordered phase of $C_{60}$ below $260 \mathrm{~K}$. The molecule is chosen to be at the origin of the space group $\mathrm{Pa} \overline{3}$. The unique $\overline{3}$ direction is [111]; reorientation may occur about one of three symmetry-equivalent [110], [011], [101] directions. The arrows indicate the sense of the rotation and the magnitude of reorientation is $\sin ^{-1}(2 / 3)=$ 41.810 . In the low-temperature phase, reorientation always occurs between major to minor orientations. The degeneracy of the three $\langle i 10\rangle$ directions ensures that all hexagons may face along the unique uniaxial [111] and that the molecular 3-fold and lattice site symmetry 3-fold axes are coincident. Reorientation about $\langle i 10\rangle$ directions also ensures that two intermolecular bonding contacts are never broken. This is an appealing possibility in the high-temperature phase for two reasons. First, comparison of high-and low-temperature orientation distribution functions indicates that the major and minor orientations still represent preferred orientations in the high-temperature phase (compare Figs. 1a and 4). Secondly, if the sense of rotation about [110] is changed when the minor orientation is facing (111) then an angular hop of only $\cos ^{-1}(1 / 3)-\sin ^{-1}(1 / 3)=$ $28.72^{\circ}$ changes the unique 3 -fold axis to [111], thus producing orientational disorder.
Fig. 2 - The atom-atom configuration for $C_{60}$ between neighbouring molecules projected along [110]. (a, upper) The more energetically favourable, major orientation involves the facing of a pentagon of "single" bonds towards one "double" bond in the next molecule; ( $b$, lower) the less energetically favourable, minor orientation involves the facing of a hexagon of mixed "single" and "double" bond character.

$m \overline{3} m\left(\mathrm{O}_{h}\right)$, to (uniaxial) trigonal, $\overline{3}\left(\mathrm{~S}_{6}\right)$. Different bucky-balls are rotated about different $<111>$ directions which are related to one another by a glide of the spacing $a$ of the lattice distance for the space group $\mathrm{Pa} \overline{3}$. Looking along the [110] bonding direction (Fig. $2 a)$, it is clear that a pentagon of one $C_{60}$ molecule faces a "double" bond of a neighbouring $\mathrm{C}_{60}$ molecule. Indeed the $6: 6$ bond site sits directly above the centre of the pentagonal face (the dihedral angle is $179.6^{\circ}$ ). Electron-poor pentagons face electron-rich "double" bonds. Alternatively, it may be considered that, in addition to the van der Waals interaction, there is an electrostatic repulsion between the "double" bond of a neighbouring molecule and the five interpentagonal electron-rich "double" bonds that surround the pentagon. This single bonding motif characterises the $\mathrm{C}_{60}-\mathrm{C}_{60}$ interactions in this model of the crystal structure.

The model is, however, an oversimplification. It assumes an ordered configuration at $5 \mathrm{~K}$ and indeed at all temperatures up to the $260 \mathrm{~K}$ first-order phase transition from primitive to face-centred cubic symmetry. NMR [6] and muon spectroscopy [7] indicate rapid reorientation below $260 \mathrm{~K}$ down to around $100 \mathrm{~K}$. So there is an apparent dilemma posed by the requirement that the $\mathrm{C}_{60}$ molecular site symmetry must possess uniaxial three-fold symmetry alone while NMR and muon measurements demand rapid quasi-isotropic reorientation. It was resolved using high resolution neutron powder diffraction experiments [8] on the basis of hopping between two distinct orientations with similar energies. In the second, less energetically favourable configuration, a hexagon faces the "double" bond of the next nearest neighbour (Fig. 2b). The energy difference between these two configurations has been derived using a number of techniques including neutron powder diffraction.

Quasi-isotropic reorientational motion has been rationalised within a uniaxial environment [8] and is illustrated in Fig. 3.

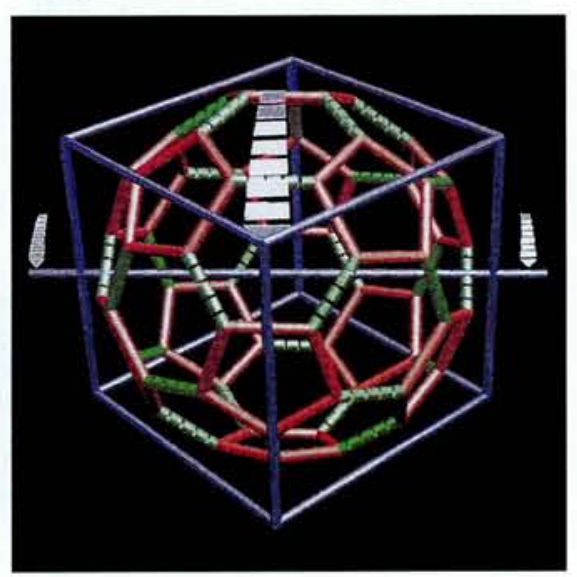

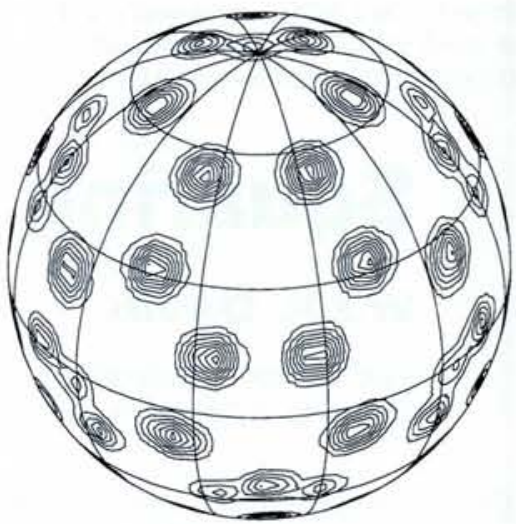

Fig. 4 - The orientation distribution function, viewed down the unique $\overline{3}$-fold [111] direction, for the orientationally ordered $C_{60}$ phase at $230 \mathrm{~K}$ as determined from high-resolution neutron powder diffraction data [4]. The fractions of major and minor orientations were $0.609(6)$ and $0.391(6)$, respectively. Ten contours are plotted from a baseline of zero scattering density.

\section{High Spin to No Spin}

There is, however, one final twist to the structure of $\mathrm{C}_{60}$ at low temperatures. An orientational glass transition occurs at around $86 \mathrm{~K}$. The anomaly manifests itself as a pronounced hump in the lattice constant, but more obviously in the temperature variation of the major orientation fraction as a freezing-in, of glass transition temperature proportions, of major and minor orientations at all temperatures below $86 \mathrm{~K}$. The kinetic nature of the $86 \mathrm{~K}$ glass transition was confirmed by specific heat [9] and more recently by single crystal dilatometry measurements [10]. Direct confirmation of the kinetics of the glass transition may also be determined from the shape of a hump in lattice constant data.

The existence of a glass transition is rather remarkable considering the rapid reorientational behaviour of $\mathrm{C}_{60}$ at room temperature. In a temperature drop of around $200 \mathrm{~K}, \mathrm{C}_{60}$ has gone from spinning faster in the solid than in solution to a kinetically forbidden orientation; a fifteen orders-ofmagnitude increase in reorientational correlation time, from an orientationally-melted to an orientational glass configuration - from high spin to no spin.

[1] Taylor R., et al., J. Chem. Soc.: Chem. Commun. No. 20 (1990) 1423

[2] Johnson R.D., et al., J. Amer. Chem. Soc. 112 (1990) 8983.

[3] Chow P.C., et al., Phys. Rev. B 69 (1992) 2943

[4] David W.I.F., et al., Proc. Roy. Soc. A (in press).

[5] Heiney P.A., et al., Phys. Rev. Lett. 66 (1991) 2911.

[6] Johnson R.D., et al., Science 255 (1992) 1235.

[7] Kiefl R.F., et al., Phys. Rev. Lett. 68 (1992) 1347.

[8] David W.I.F., et al., Europhys. Lett. 18 (1992) 219

[9] Matsuo T., et al., Solid State Commun. 83 (1992) 711

[10] Gugenberger F., et al., Phys. Rev. Lett. 69 (1992) 3774 